\title{
Comparing fluorescence-based cell-free assays for the assessment of antioxidative capacity of high-density lipoproteins
}

\author{
Fumiyoshi Tsunoda, Stefania Lamon-Fava, Katalin V. Horvath, Ernst J. Schaefer and Bela F. Asztalos*
}

\begin{abstract}
Background: Population studies have shown an inverse association between high-density lipoprotein (HDL) cholesterol levels and risk of coronary heart disease (CHD). HDL has different functions, including the ability to protect biological molecules from oxidation. Our aim was to evaluate the performance of two fluorescence-based assays in assessing the antioxidative capacity of $\mathrm{HDL}$.

Methods: We compared the antioxidative capacity of HDL with the phospholipid 2',7'-dichlorodihydrofluorescein (DCF) assay and the dihydrorhodamine 123 (DHR) assay in controls and in subjects at increased risk of CHD, including subjects with established CHD, and subjects with elevated plasma triglycerides (TG), serum amyloid A (SAA), or myeloperoxidase (MPO) levels.

Results: The antioxidative capacity of HDL, as measured by the DCF assay, was significantly lower in both CHD and high-TG patients than in controls ( $p<0.001$ and $p=0.004$, respectively). Interestingly, the mean antioxidative capacity of HDL in high-SAA subjects was significantly higher $(p<0.03)$, while in high-MPO subjects was similar to controls. When the DHR assay was used we did not find differences in HDL's antioxidative capacity between CHD patients and controls but we found higher antioxidative capacity in high-SAA subjects compared to controls.

Conclusions: Only the DCF assay could detect significant differences in the antioxidative capacity of HDL between controls and CHD subjects. Practical use of both assays for the assessment of antioxidative capacity of HDL is limited by the large overlap in values among groups. The antioxidative activity of HDL in patients who have elevated SAA levels needs to be reassessed.
\end{abstract}

Keywords: High density lipoprotein, Oxidation, Coronary heart disease, Fluorescence assay

\section{Background}

The epidemiological evidence of an inverse association between plasma high-density lipoprotein cholesterol (HDL-C) levels and risk of cardiovascular disease is well established [1, 2]. HDL can protect against the development and progression of atherosclerosis by promoting reverse cholesterol transport (RCT), maintaining endothelial function, and reducing inflammation and oxidation [3]. In subjects who had undergone assessment of cardiovascular disease by coronary angiography or carotid intima-media thickness, the cholesterol efflux capacity of HDL was

\footnotetext{
* Correspondence: bela.asztalos@tufts.edu

From the Cardiovascular Nutrition Laboratory, Jean Mayer USDA Human Nutrition Research Center on Aging, Tufts University, 711 Washington Street, Boston, MA 02111, USA
}

shown to be inversely associated with the extent of cardiohas been observed [7]. Therefore, it is now suggested that measurement of HDL functional capacity may be a better predictor of cardiovascular disease risk than HDL-C levels. Navab et al. [8] and Kelesidis et al. [9] have developed cell-free assays to measure the antioxidative activity of $\mathrm{HDL}$, one of the functional capacities of HDL, using fluorescence probes. A number of studies using these assays 
have shown an impaired antioxidative activity of HDL in patients with both acute and chronic inflammatory disorders such as acute coronary syndrome (ACS), systemic lupus erythematosus, and diabetes $[5,6,10]$. In these studies, although the assays could detect differences between control subjects and patients, there was considerable overlap between the groups and large standard deviation of the mean values.

To validate the performance of the fluorescence probebased antioxidative assays, we sought to determine whether these assays could detect differences between apparently healthy subjects and patients whose conditions are thought to impair the antioxidative activity of HDL.

\section{Methods}

\section{Subjects and samples}

Venous blood samples were collected from 160 apparently healthy subjects (controls), 98 patients with cardiovascular disease, 37 subjects with elevated triglyceride (TG) levels (>250 mg/dL), 40 subjects with elevated serum amyloid A (SAA) levels ( $>300 \mathrm{mg} / \mathrm{L})$, and 30 subjects with elevated serum myeloperoxidase (MPO) levels ( $>500 \mathrm{pmol} / \mathrm{L})$. Mean age of control and CHD subjects was $58 \pm 16$ and $61 \pm 7$ years, respectively. Control subjects had normal plasma total cholesterol (TC), TG and low-density lipoprotein cholesterol (LDL-C) levels (less than 200, 150, and $160 \mathrm{mg} / \mathrm{dL}$, respectively). Subjects with cardiovascular disease had established coronary heart disease (CHD), defined as history of previous MI, stable angina, a catheterization report of $\geq 50 \%$ stenosis of at least one coronary artery, abnormal exercise tolerance test or nuclear imaging procedure, or angioplasty. Hypertension was present in $4.5 \%$ of controls and in $48 \%$ of CHD subjects and type 2 diabetes in $3.4 \%$ of controls and $19 \%$ of $\mathrm{CHD}$ subjects. Also, hypertension was present in $13 \%$ of high TG, $10 \%$ of high SAA and $11 \%$ of high MPO subjects, while diabetes was present in $9 \%$ of high TG, $10 \%$ of high SAA and $6 \%$ of high MPO subjects.

Plasma and serum samples were obtained by centrifugation and immediately stored at $-80{ }^{\circ} \mathrm{C}$ until measurement. Only plasma or serum were available in some study subjects, therefore, appropriate plasma and serum controls were used. The HDL antioxidative activity was measured in different biological samples: HDL fractions obtained by ultracentrifugation (UCHDL), apoB-depleted (apoBd) plasma, or apoBd serum. ApoBd plasma (or serum) samples were obtained by adding 0.4 parts of a polyethylene glycol (PEG) 6000 solution (Qiagen, Valencia, CA) $(20 \%$ PEG in $200 \mathrm{mM}$ glycine buffer, $\mathrm{pH}$ 7.4) to 1 part plasma (or serum) followed by centrifugation at $10,000 \mathrm{~g}$ for $30 \mathrm{~min}$ at $4{ }^{\circ} \mathrm{C}$. ApoBd samples were assayed within one hour. UCHDL samples were separated by density-gradient ultracentrifugation $($ density $=1.063-1.210 \mathrm{~g} / \mathrm{mL}$ ) followed by dialysis at $4{ }^{\circ} \mathrm{C}$. UCHDL samples were assayed within three hours after dialysis.

\section{Laboratory measurements}

Total cholesterol, low-density lipoprotein cholesterol (LDL-C), HDL-C, TG, and apoA-I levels were measured using automated standardized assays from Roche (Indianapolis, IN). Small-dense LDL-C (sdLDL-C) levels were measured using kits provided by Denka-Seiken (Tokyo, Japan) [11]. SAA and MPO levels were measured using kits from Siemens on a Dimension and a BNII clinical chemistry system, respectively. The apoAI-containing HDL subpopulation profile was determined by non-denaturing two-dimensional gel electrophoresis as previously described [12]. High sensitivity $C$ reactive protein (hs-CRP), glucose and insulin levels were measured in a central laboratory using established assays.

\section{Measurement of the antioxidative activity of HDL}

The antioxidative activity of HDL was measured by two different fluorescence-based cell-free assays.

The antioxidant capacity of HDL was measured in 269 subjects by Vascular Strategies (Plymouth Meeting, PA) using the organic phospholipid 2,7'-dichlorodihydrofluorescein (DCF) diacetate probe, as previously described by Navab et al. [8]. This assay is based on the inhibition of oxidation of the fluorescence probe by HDL. Briefly, apoBd plasma or serum samples were incubated with oxidized LDL and DCF diacetate. In the presence of oxidized LDL, DCF diacetate was converted to DCF, its fluorescence form. The fluorescence excitation/emission was measured at a $485 / 528 \mathrm{~nm}$ for one hour. To calculate the antioxidant value of HDL of each sample, the fluorescence intensity was divided by a reference value. This ratio is commonly referred to as the HDL inflammatory index (HII) [8]. A value $>1.0$ indicates dysfunctional (pro-oxidant) HDL, while values < 1.0 indicate normal (antioxidant) HDL. The second assay was used in our laboratory to determine the antioxidative activity of HDL using the dihydrorhodamine 123 (DHR) as the fluorescence probe [9]. DHR (Life Technologies, Gaithersburg, MD) was dissolved in dimethyl sulfoxide (DMSO) to a $50 \mathrm{mM}$ DHR stock concentration. Iron-free buffered saline was prepared as follows: $60 \mathrm{nM}$ of deferoxamine mesylate (Sigma-Aldrich, Saint Louis, MO) was added to HBS buffer (HEPES $20 \mathrm{mM}$, $\mathrm{NaCl} 150 \mathrm{mM}, \mathrm{pH}$ 7.4) followed by treatment with $1.0 \mathrm{~g} / \mathrm{dL}$ of Chelex 100 resin (Bio-Rad, Hercules, CA), as previously described [13]. The concentrated DHR stock reagent was diluted 1:1000 in the iron-free HBS buffer to the $50 \mu \mathrm{M}$ DHR working solution just before use. Five $\mu \mathrm{g}$ of HDL-C of either UCHDL or apoBd samples was added to each well in a 96-well flat bottom black plate (Fisher Scientific, Waltham, MA). The volume of each well was 
adjusted to $150 \mu \mathrm{L}$ using the iron-free HBS buffer. Then, $25 \mu \mathrm{L}$ of the $50 \mu \mathrm{M}$ DHR solution was added to reach a final DHR concentration of $7 \mu \mathrm{M}$ and the plate was immediately placed into an FLx800 Fluorescence Reader (BioTek, Winooski, VT). Samples were run in quadruplicate and a standard sample was added to each assay plate. The fluorescence intensity of oxidized DHR was assessed at five-minute intervals for one hour at $485 / 528 \mathrm{~nm}$ excitation/emission. The oxidative rate of DHR (ORD) was calculated as the linear regression slope of the fluorescence intensity between 10 and $60 \mathrm{~min}$ as calculated with Microsoft Excel software. The ORD value of each sample was determined as the mean of the quadruplicate slopes.

For both assays, the lower the number, the higher the antioxidant capacity of HDL.

\section{Statistical analysis}

Statistical analyses were performed with the SPSS software version 22 (IBM SPSS, IBM Corporation, Somers, NY). Variables with normal distribution were expressed as mean \pm SD and those with non-normal distribution were expressed as median and interquartile ranges. Differences between values among groups were tested by Mann-Whitney $U$ test. Spearman correlation coefficients describe the relationships between variables. Statistical significance was accepted at $p<0.05$.

\section{Results}

Antioxidant capacity of HDL as measured by the DCF assay Analysis of antioxidative capacity of apoB-depeted (apoBd) samples, as assessed with the DCF assay, in control subjects revealed no significant differences between men and women (Table 1). Therefore, in subsequent analyses, men and women were combined in one group. However, serum samples had significantly lower antioxidative capacity than plasma samples (Table 1); therefore, in our analyses we matched for sample type.

Characteristics of control subjects and patients whose DCF values were measured in apoBd plasma or serum are described in Table 2. All the lipid parameters in CHD patients and high TG subjects were significantly different from those in controls, except for apoA-I levels which were similar in controls and CHD patients. The CHD, high TG, and high SAA subjects had significantly higher TG levels than controls, and this was associated with lower HDL-C levels and altered distribution of HDL particles. Median DCF values in both CHD patients and high TG patients were significantly higher (indicating reduced antioxidative activity of HDL) than in controls $(p<0.001$ and $p=0.004$, respectively) (Fig. 1). Although high SAA subjects had lower HDL-C and higher TG levels than controls, their mean DCF value was significantly lower (better antioxidative activity of HDL) than that of controls $(p=0.03)$ (Fig. 1). In subjects with high MPO, serum lipids were similar to those of controls, with the exception of HDL-C (Table 2). The antioxidative capacity of $\mathrm{HDL}$ as measured in apoBd serum in high MPO subjects was similar to that of controls $(0.356 \pm 0.035$ and $0.378 \pm 0.056$, respectively).

\section{Correlations between HDL subpopulations and HDL antioxidative capacity}

HDL's antioxidative capacity was inversely associated with the relative (\%) concentrations of the small pre $\beta-1$ and $\alpha-3$ HDL particles $(r=-0.162, p=0.030$, and $r=-0.155, p=$ 0.037 , respectively), and was positively associated with HDL-C concentrations and percent distribution of the

Table 1 Plasma lipid levels and antioxidative capacity of HDL as measured by the DCF assay in apoBd plasma and serum samples in control subjects

\begin{tabular}{|c|c|c|c|c|}
\hline & \multicolumn{4}{|c|}{ Control Subjects } \\
\hline & \multicolumn{2}{|l|}{ Plasma } & \multicolumn{2}{|l|}{ Serum } \\
\hline & Men & Women & Men & Women \\
\hline N & 30 & 29 & 32 & 25 \\
\hline Men/women & $30 / 0$ & $0 / 30$ & $32 / 0$ & $0 / 25$ \\
\hline Age, y & $59 \pm 17$ & $56 \pm 16$ & $56 \pm 18$ & $59 \pm 14$ \\
\hline $\mathrm{TC}, \mathrm{mg} / \mathrm{dL}$ & $157 \pm 28$ & $175 \pm 24$ & $185 \pm 39$ & $189 \pm 38$ \\
\hline $\mathrm{HDL}-\mathrm{C}, \mathrm{mg} / \mathrm{dl}$ & $53 \pm 11$ & $67 \pm 13^{*}$ & $53 \pm 13$ & $68 \pm 15^{*}$ \\
\hline $\mathrm{LDL}-\mathrm{C}, \mathrm{mg} / \mathrm{dL}$ & $93 \pm 21$ & $95 \pm 17$ & $117 \pm 36$ & $103 \pm 27$ \\
\hline $\mathrm{TG}, \mathrm{mg} / \mathrm{dL}$ & $87 \pm 37$ & $78 \pm 23$ & $102 \pm 30$ & $95 \pm 39$ \\
\hline $\mathrm{sdLDL}-\mathrm{C}, \mathrm{mg} / \mathrm{dL}$ & $17 \pm 7$ & $19 \pm 8$ & $27 \pm 8$ & $25 \pm 12$ \\
\hline Apo A-l, mg/dL & $148 \pm 19$ & $170 \pm 25$ & $154 \pm 28$ & $182 \pm 28$ \\
\hline Antioxidative capacity of HDL (HII) & $0.28 \pm 0.04$ & $0.30 \pm 0.05$ & $0.38 \pm 0.06^{* *}$ & $0.38 \pm 0.05^{* *}$ \\
\hline
\end{tabular}

${ }^{*} p<0.05$, compared to men

${ }^{* *} p<0.05$ compared to plasma 
Table 2 Plasma or serum lipid profiles in control subjects and patients whose HDL antioxidative activities were measured in apoBd plasma or serum, respectively, with the DCF assay

\begin{tabular}{|c|c|c|c|c|c|c|}
\hline & \multicolumn{4}{|l|}{ Plasma } & \multicolumn{2}{|l|}{ Serum } \\
\hline & Controls & $\mathrm{CHD}$ & High TG & High SAA & Controls & High MPO \\
\hline Number & 59 & 58 & 37 & 28 & 57 & 30 \\
\hline$M / F, n$ & $30 / 29$ & $54 / 4^{* *}$ & $25 / 12$ & $19 / 9$ & $32 / 25$ & $15 / 15$ \\
\hline $\mathrm{TC}, \mathrm{mg} / \mathrm{dL}$ & $165 \pm 26$ & $145 \pm 29^{* *}$ & $262 \pm 178^{* *}$ & $163 \pm 44$ & $188 \pm 38$ & $169 \pm 46$ \\
\hline $\mathrm{HDL}-\mathrm{C}, \mathrm{mg} / \mathrm{dL}$ & $59 \pm 14$ & $43 \pm 13^{* *}$ & $34 \pm 15^{* *}$ & $51 \pm 17^{*}$ & $60 \pm 15$ & $49 \pm 16^{*}$ \\
\hline $\mathrm{LDL}-\mathrm{C}, \mathrm{mg} / \mathrm{dL}$ & $94 \pm 19$ & $80 \pm 23^{* *}$ & $115 \pm 68^{*}$ & $89 \pm 6$ & $112 \pm 35$ & $100 \pm 41$ \\
\hline $\mathrm{TG}, \mathrm{mg} / \mathrm{dL}$ & 74 (63-99) & $128(88-170)^{* *}$ & $324(264-1080)^{* *}$ & $115(92-163)^{*}$ & $97(70-123)$ & $116(93-146)$ \\
\hline $\mathrm{sd}$ LDL-C, mg/dL & 16 (14-19) & $37(27-44)^{* *}$ & $41(27-63)^{* *}$ & $17(12-27)$ & $25(18-32)$ & $23(19-30)$ \\
\hline Apo A-I, mg/dL & $158 \pm 23$ & $152 \pm 34$ & $129 \pm 45^{* *}$ & $148 \pm 48$ & $168 \pm 29$ & $149 \pm 33$ \\
\hline Glucose, mg/dL & $110 \pm 17$ & $103 \pm 31$ & $164 \pm 80$ & $110 \pm 21$ & $92 \pm 15$ & $105 \pm 23$ \\
\hline Insulin $\mu \mathrm{IU} / \mathrm{mL}$ & $13.2 \pm 13.1$ & $15.1 \pm 9.5$ & $19.8 \pm 12.1$ & $14.1 \pm 7.2$ & $8.3 \pm 5.1$ & $22.0 \pm 13.5$ \\
\hline $\mathrm{Hs}-\mathrm{CRP}, \mathrm{mg} / \mathrm{L}$ & $1.7 \pm 1.9$ & $2.4 \pm 4.9$ & $7.9 \pm 15.1$ & $5.5 \pm 4.4$ & $1.3 \pm 1.2$ & $2.7 \pm 11.6$ \\
\hline \multicolumn{7}{|l|}{ HDL distribution } \\
\hline Preß-1, \% & $8(6-10)$ & $12(9-15)^{* *}$ & $15(10-19)^{* *}$ & $8(6-11)$ & $5(4-7)$ & $5(4-7)$ \\
\hline$a-1, \%$ & $23(20-28)$ & $15(12-19)^{* *}$ & $11(7-17)^{* *}$ & $17(12-21)^{* *}$ & $22(19-25)$ & $20(16-22)$ \\
\hline$a-2, \%$ & $44(42-47)$ & $42(39-45)^{*}$ & $41(36-44)^{*}$ & $54(49-61)^{* *}$ & $41(37-44)$ & 40 (39-43) \\
\hline$a-3, \%$ & $15(13-16)$ & $18(16-20)^{* *}$ & $17(13-23)^{* *}$ & $11(9-15)^{* *}$ & $13(12-15)$ & $15(13-17)$ \\
\hline$a-4, \%$ & $10(8-11)$ & $12(10-14)^{* *}$ & $13(10-16)^{* *}$ & $6(5-9)^{* *}$ & $11(9-12)$ & $12(10-14)$ \\
\hline
\end{tabular}

Data are shown as mean \pm SD or median (interquartile range). Differences in values between each group and Controls were tested by Pearson chi-squared test, Student- $t$ test or Mann-Whitney's $U$ test $\left({ }^{*} p<0.05\right.$ and ${ }^{* *} p<0.01$, relative to Controls)

\section{Antioxidant capacity of HDL (DCF assay) in controls and high CHD risk subjects}

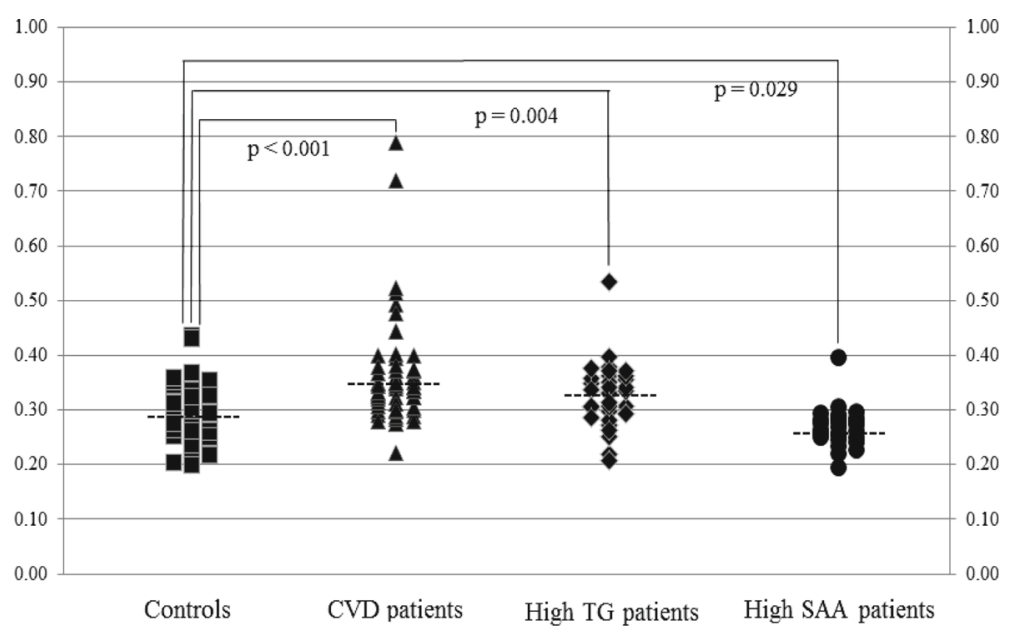

Fig. 1 Distribution and median values of the antioxidant capacity of HDL as assessed by the DCF assay in apoBd plasma samples of apparently healthy subjects (Controls), patients with cardiovascular disease (CVD patients), patients with high triglyceride levels (High TG patients), and patients with high serum amyloid A levels (High SAA patients). Differences between groups were tested by Mann-Whitney $U$ test 
intermediate size $\alpha-2$ HDL particles $(r=0.198 p=0.008$ and $r=0.246, p=0.001$, respectively) (Table 2 ).

\section{Antioxidant capacity of HDL as measured by the DHR assay}

Plasma lipid profiles of the subjects whose oxidation rate (ORD) were measured with the DHR assay are shown in Table 3. HDL-C and apoA-I levels were lower in CHD patients than in controls. Plasma lipid profiles of high-SAA subjects were not significantly different from controls. ORD was measured using both UCHDL and apoBd plasma in control and CHD subjects. The median level of HDL antioxidative capacity was similar in controls and CHD patients, whether measured using UCHDL or apoBd plasma samples (Fig. 2). However, the antioxidant capacity was significantly higher in UCHDL than in apoBd plasma (both $p<0.001$ ) (Fig. 2). High SAA subjects had significantly lower median ORD value (better antioxidative activity of HDL) compared to controls. Unexpectedly, there was an inverse association between the ORD values obtained from UCHDL and apoBd plasma (Spearman correlation coefficient: $-0.264, p=0.024$ ) (Fig. 3).

\section{Discussion}

It has been proposed that HDL can protect against atherosclerosis by reducing LDL oxidation and protecting cells from oxidative stress, important players in the development and progression of atherosclerotic lesions [14]. A number of studies using the DCF assay have demonstrated that patients with chronic inflammatory diseases have lower antioxidative capacity (higher DCF values, expressed as HII) compared to healthy subjects [15-17]. Recently, it has also been reported that higher HII values are associated with increased mortality in critically ill patients [18]. On the other hand, another study has shown that, while acute coronary syndrome

Table 3 Plasma lipid profiles of control subjects and patients whose HDL antioxidative capacity was measured in ultracentrifuged $\mathrm{HDL}$ (UCHDL) and apoBd plasma samples using the DHR assay

\begin{tabular}{llll}
\hline & Controls & CHD & High SAA \\
\hline Number & 41 & 40 & 12 \\
M/F, n & $14 / 27$ & $22 / 18$ & $7 / 5$ \\
TC, mg/dL & $174 \pm 36$ & $171 \pm 41$ & $193 \pm 79$ \\
HDL-C, mg/dL & $66 \pm 18$ & $53 \pm 17^{* *}$ & $72 \pm 58$ \\
LDL-C, mg/dL & $102 \pm 27$ & $99 \pm 36$ & $99 \pm 44$ \\
TG, mg/dL & $93(68-122)$ & $103(77-153)$ & $84(62-133)$ \\
sd LDL-C, mg/dL & $23(17-29)$ & $38(23-45)$ & $27(17-32)$ \\
Apo A-l, mg/dL & $172 \pm 29$ & $156 \pm 33^{*}$ & $168 \pm 78$ \\
\hline
\end{tabular}

Data shown as mean \pm SD or median (interquartile range). $P$ value of the maleto-female ratio between the patients with cardiovascular disease and the control subjects is 0.059 analyzed by chi-squared test

${ }^{*} p<0.05,{ }^{* *} p<0.01$ compared to controls patients had significantly higher than normal HII values, no differences were observed between chronic CHD patients and healthy subjects [5]. These results suggest that the DCF assay can detect alterations in the antioxidative potential of HDL in some, but not all patient populations.

Similarly, in our study, we have shown that the antioxidative capacity of HDL assessed by the DCF assay is significantly lower in CHD patients (higher HII) than in healthy subjects. High TG levels were also associated with decreased antioxidant capacity. However, it should be noted that none of the CHD subjects in our study had a DCF value, or HII index, above 1 , a set limit of pro-inflammatory HDL. Moreover, other conditions that are believed to be associated with dysfunctional HDL, such as elevated SAA or MPO levels were not associated with impaired antioxidative capacity [19]. Actually, subjects with elevated SAA levels showed a higher antioxidant capacity than controls. Similarly to other studies, our study documented a large overlap in the measures of antioxidative activities of HDL among controls and diseased subjects.

HDL particles are heterogeneous in size, composition and function [12]. A number of studies have revealed that various HDL subpopulations are differently associated with the antioxidative activity of HDL. Kontush et al. [20, 21] demonstrated that in normolipidemic subjects, the smaller HDL3 fraction had more favorable biological and functional properties than the larger HDL2 fraction and that the antioxidative activity of HDL was predominantly associated with HDL3. Other researchers have shown that, in diabetic patients, unfavorable changes in the chemical composition and enzyme activities of HDL3 are associated with reduced antioxidative activity of HDL $[22,23]$. Our data indicating a significant correlation between and HDL-C and HDL antioxidant capacity is in line with others' data [5]. When we measured HDL subpopulations, we observed an inverse correlation between DCF and $\alpha-2$ HDL particles. This HDL subpopulation is found mostly in the HDL3 fraction [23]. Therefore, our findings are in agreement with previous studies and support a specific involvement of the normally most abundant HDL particle, $\alpha-2$ in the antioxidative capacity of HDL. Interestingly, LDL-C levels also correlated inversely with DCF value: it is possible that statin use in the CHD patients explains this unexpected result, since statin therapy has been previously shown to improve DCF values [5]. In contrast to total LDL-C, sdLDL-C, the most atherogenic fraction of LDL, had a positive correlation with DCF values.

Our data also indicate that DCF values were higher in serum than in plasma samples. This difference may be explained, at least in part, by the antioxidant effects of fibrinogen [24]. This raises the question whether we can measure HDL's antioxidative capacity in apoBd samples 
Antioxidant capacity of HDL (ORD) in Ultracentrifuge HDL and apoB depleted plasma samples

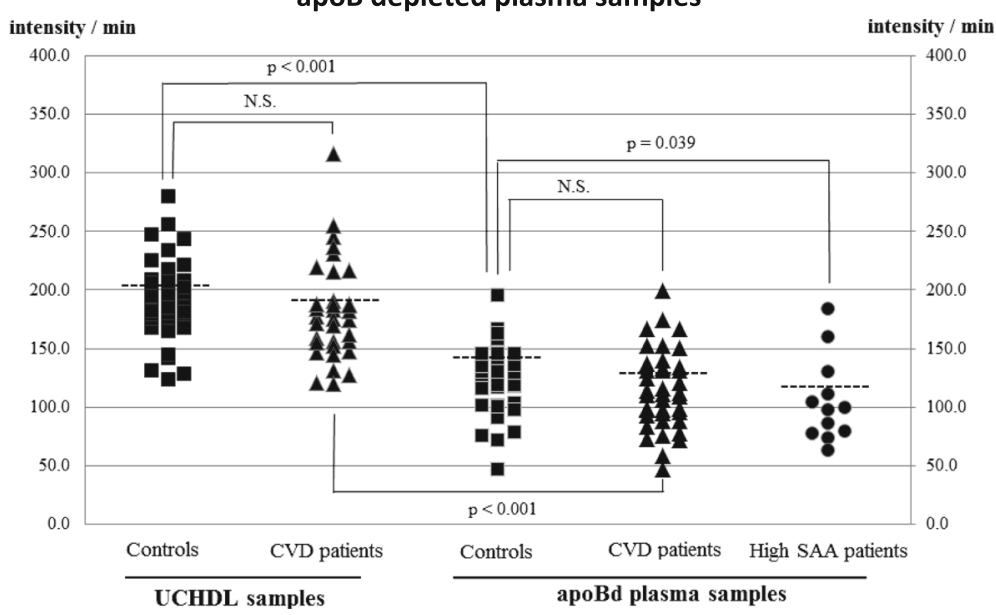

Fig. 2 Distribution and median values of the oxidation rate of DHR (ORD) of ultracentrifuged HDL fractions (UCHDL) from apparently healthy subjects (Controls) and patients with the cardiovascular disease (CVD patients), and of apoB-deleted (apoBd) plasma from Controls, CVD patients, and patients with high serum amyloid A protein levels (High SAA patients). Differences between groups were tested by Mann-Whitney $U$ test

which contains a large number of other potentially redox compounds. On the other hand, it is well documented that HDL goes through significant alteration during ultracentrifugal separation, which might alter its redox capacity.
When the DHR assay was used to measure HDL antioxidative capacity, we did not observe a significant difference between the median values in controls and CHD patients. A previous study using the DHR assay has shown that, although HDL from patients with psoriasis

\section{Correlation between ORD values using UCHDL and apoBd plasma}

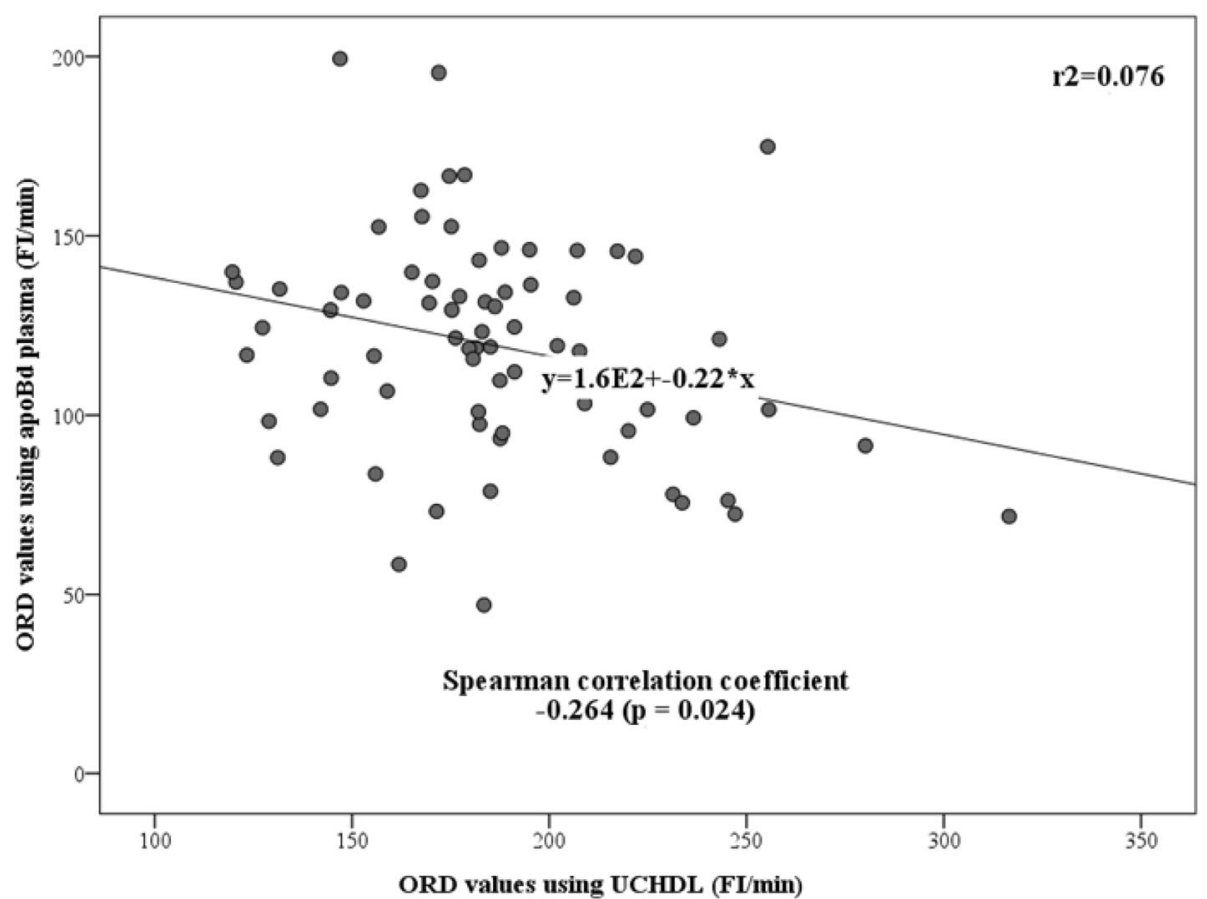

Fig. 3 Correlation between the oxidation rate of DHR (ORD) of ultracentrifuged HDL fraction (UCHDL) and of apoB-depleted (apoBd) plasma in apparently healthy subjects and patients with cardiovascular disease 
had lower cholesterol efflux capacity than control subjects, there was no difference in the HDL antioxidant capacity between the two groups [25]. Kelesidis et al. [26], who had developed the DHR assay, have described some of the limitations of the assay and reported that the ORD value is susceptible to not only environmental factors but also to factors associated with lipoproteins, such as type and concentration of each lipoprotein particle, profile of apolipoproteins, and lipoprotein-isolation methods. For example, the weight of added LDL, a proinflammatory lipoprotein particle, correlated significantly and inversely with the DHR value. Moreover, in our study, the median ORD value from UCHDL samples was significantly higher than that of apoBd samples: furthermore, the ORD values of UCHDL samples correlated negatively with those of apoBd samples. Similarly, Kelesidis et al. have observed differences between UCHDL and apoBd serum values, as indicated by median ORD value in patients infected by HIV being significantly higher than in healthy subjects when using apoBd serum samples but lower when using UCHDL samples [26]. Based on these data and the results of our study, DHR should be considered an unsuitable fluorescent probe for the measurement of the antioxidative activity of HDL.

SAA, an acute-phase protein primarily synthesized by hepatocytes, replaces apoA-I in HDL and forms monodisperse $(\alpha-2)$ HDL particles. A number of experimental studies have suggested that SAA can accelerate atherosclerosis possibly by impairing the antioxidative capacity of HDL $[27,28]$. In contrast, our study indicated that patients with high SAA levels had significantly lower ORD or DCF values (better antioxidative activity of $\mathrm{HDL}$ ) than controls.

A limitation of our study was that we did not have complete information on medical histories and medications in all of our study subjects. Another potential limitation is that the number of the subjects might be too small to yield significant results about the DHR assay.

\section{Conclusions}

We are led to the following conclusions: 1) the antioxidant capacity of HDL is affected by the medium: apoB-depleted plasma and serum, and ultracentrifugally isolated HDL exert different antioxidant capacity; 2 ) although the DCF assay could detect significant differences in the antioxidative activity of HDL between controls and patients, the practical use of this assay is limited by the large overlap in values among groups; 3) the DHR assay failed to measure accurately the antioxidative activity of HDL, possibly due to the susceptibility of the probe to various factors; and 4) the antioxidative activity of HDL in patients who have elevated SAA levels needs to be reassessed.

\section{Acknowledgments}

We would like to thank our laboratory staff for their assistance in the measurements of plasma lipid, MPO and SAA levels.

\section{Funding}

The study was supported by a grant from Boston Heart Diagnostics, by NIH/ NHLBI grant R01HL117933 to BFA, and USDA agreement No. 58-1950-0-0014. The contents of this manuscript are solely the responsibility of the authors and do not necessarily represent the official views of the National Institutes of Health or the US Department of Agriculture.

Availability of data and material

Please contact corresponding author for reasonable data requests.

\section{Authors' contributions}

All authors contributed to designing the experiment, interpreting the data and preparing, revising and approving the final version of the manuscript. FT processed and analyzed the data. All authors have read and approved the final manuscript.

\section{Competing interests}

Bela Asztalos is a consultant for and receives regular compensation from Boston Heart Diagnostics. Ernst J Schaefer and Katalin Horvath are part time employees of Boston Heart Diagnostics.

\section{Consent for publication}

Not applicable.

\section{Ethics approval}

The study was reviewed by the Tufts Health Sciences Institutional Review Board and granted exempt status because only de-identified samples were analyzed in the study.

Received: 28 May 2016 Accepted: 14 September 2016 Published online: 22 September 2016

\section{References}

1. Gordon T, Castelli WP, Hjortland MC, Kannel WB, Dawber TR. High density lipoprotein as a protective factor against coronary heart disease. The Framingham Study. Am J Med. 1977;62:707-14.

2. Gordon DH, Rifkind BM. High-density lipoprotein: the clinical implications of recent studies. N Engl J Med. 1989;321:1311-6.

3. Rosenson RS, Brewer Jr HB, Ansell B, Barter P, Chapman MJ, Heinecke JW, et al. Translation of high-density lipoprotein function into clinical practice: current prospects and future challenges. Circulation. 2013;128(11):1256-67.

4. Khera AV, Cuchel M, de la Llera-Moya M, Rodrigues A, Burke MF, Jafri K, et al. Cholesterol efflux capacity, high-density lipoprotein function, and atherosclerosis. N Engl J Med. 2011;364(2):127-35.

5. Patel PJ, Khera AV, Jafri K, Wilensky RL, Rader DJ. The anti-oxidative capacity of high-density lipoprotein is reduced in acute coronary syndrome but not in stable coronary artery disease. J Am Coll Cardiol. 2011;58(20):2068-75.

6. Morgantini C, Natali A, Boldrini B, Imaizumi S, Navab M, Fogelman AM, et al. Anti-inflammatory and antioxidant properties of HDLs are impaired in type 2 diabetes. Diabetes. 2011;60(10):2617-23.

7. de la Llera Moya M, McGillicuddy FC, Hinkle CC, Byrne M, Joshi MR, Nguyen $\checkmark$, et al. Inflammation modulates human HDL composition and function in vivo. Atherosclerosis. 2012;222(2):390-4.

8. Navab M, Hama SY, Hough GP, Subbanagounder G, Reddy ST, Fogelman AM. A cell-free assay for detecting HDL that is dysfunctional in preventing the formation of or inactivating oxidized phospholipids. J Lipid Res. 2001; 42(8):1308-17.

9. Kelesidis T, Currier JS, Huynh D, Meriwether D, Charles-Schoeman C, Reddy ST, et al. A biochemical fluorometric method for assessing the oxidative properties of HDL. J Lipid Res. 2011;52(12):2341-51.

10. Hahn BH, Lourencco EV, McMahon M, Skaggs B, Le E, Anderson M, et al. Pro-inflammatory high-density lipoproteins and atherosclerosis are induced in lupus-prone mice by a high-fat diet and leptin. Lupus. 2011;19(8):913-7.

11. Ito Y, Fujimura M, Ohta M, Hirano T. Development of a homogeneous assay for measurement of small dense LDL cholesterol. Clin Chem. 2011;57(1):57-65. 
12. Asztalos BF, Sloop CH, Wong L, Roheim PS. Two-dimensional electrophoresis of plasma lipoproteins: recognition of new apo A-l-containing subpopulations. Biochim Biophys Acta. 1993;1 169(3):291-300.

13. Esposito BP, Breuer W, Sirankapracha P, Pootrakul P, Hershko C, Cabantchik ZI. Labile plasma iron in iron overload: redox activity and susceptibility to chelation. Blood. 2003;102(7):2670-7.

14. Madamanchi NR, Vendrov A, Runge MS. Oxidative stress and vascular disease. Arterioscler Thromb Vasc Biol. 2005;25:29-38.

15. McMahon M, Grossman J, FitzGerald J, Dahlin-Lee E, Wallace DJ, Thong BY, et al. Proinflammatory high-density lipoprotein as a biomarker for atherosclerosis in patients with systemic lupus erythematosus and rheumatoid arthritis. Arthritis Rheum. 2006;54(8):2541-9.

16. van Leuven SI, Hezemans R, Levels JH, Snoek S, Stokkers PC, Hovingh GK, et al. Enhanced atherogenesis and altered high density lipoprotein in patients with Crohn's disease. J Lipid Res. 2007:48(12):2640-6.

17. Patel PJ, Khera AV, Wilensky RL, Rader DJ. Anti-oxidative and cholesterol efflux capacities of high-density lipoprotein are reduced in ischaemic cardiomyopathy. Eur J Heart Fail. 2013;15(11):1215-9.

18. Schrutka L, Goliasch G, Meyer B, Wurm R, Koller L, Kriechbaumer L, Heinz G, Pacher R, Lang IM, Distelmaier K, Hülsmann M. Impaired high-density lipoprotein anti-oxidant function predicts poor outcome in critically III patients. PLoS One. 2016;11(3):e0151706.

19. Smith JD. Myeloperoxidase, inflammation, and dysfunctional HDL. J Clin Lipidol. 2010:4:382-8.

20. Kontush A, Chantepie S, Chapman MJ. Small, dense HDL particles exert potent protection of atherogenic LDL against oxidative stress. Arterioscler Thromb Vasc Biol. 2003;23(10):1881-8.

21. Kontush A, Therond P, Zerrad A, Couturier M, Negre-Salvayre A, de Souza $J A$, et al. Preferential sphingosine-1-phosphate enrichment and sphingomyelin depletion are key features of small dense HDL3 particles: relevance to antiapoptotic and antioxidative activities. Arterioscler Thromb Vasc Biol. 2007;27(8):1843-9.

22. Nobecourt E, Jacqueminet S, Hansel B, Chantepie S, Grimaldi A, Chapman $\mathrm{MJ}$, et al. Defective antioxidative activity of small dense HDL3 particles in type 2 diabetes: relationship to elevated oxidative stress and hyperglycaemia. Diabetologia. 2005;48(3):529-38.

23. Asztalos BF, Roheim PS, Milani RL, Lefebre M, McNamara JR, Horvath KV, Schaefer EJ. Distribution of apoA-I containing HDL subpopulations in patients with coroanry heart disease. Arterioscler Thromb Vasc Biol. 2000; 20:2670-6.

24. Kaplan IV, Attaelmannan M, Levinson SS. Fibrinogen is an antioxidant that protects $\beta$-lipoproteins at physiological concentrations in a cell free system. Atherosclerosis. 2001;158:455-63.

25. Holzer M, Wolf P, Curcic S, Birner-Gruenberger $\mathrm{R}$, Weger $\mathrm{W}$, Inzinger $\mathrm{M}$, et al. Psoriasis alters HDL composition and cholesterol efflux capacity. J Lipid Res. 2012;53(8):1618-24.

26. Kelesidis T, Reddy ST, Huynh D, Meriwether D, Fogelman AM, Navab M, et al. Effects of lipid-probe interactions in biochemical fluorometric methods that assess HDL redox activity. Lipids Health Dis. 2012;11:87.

27. King VL, Thompson J, Tannock LR. Serum amyloid A in atherosclerosis. Curr Opin Lipidol. 2011;22(4):302-7.

28. Rached F, Lhomme M, Camont L, Gomes F, Dauteuille C, Robillard P, et al. Defective functionality of small, dense HDL3 subpopulations in ST segment elevation myocardial infarction: relevance of enrichment in lysophosphatidylcholine, phosphatidic acid and serum amyloid A. Biochim Biophys Acta. 2015;1851(9):1254-61.

\section{Submit your next manuscript to BioMed Central and we will help you at every step:}

- We accept pre-submission inquiries

- Our selector tool helps you to find the most relevant journal

- We provide round the clock customer support

- Convenient online submission

- Thorough peer review

- Inclusion in PubMed and all major indexing services

- Maximum visibility for your research

Submit your manuscript at www.biomedcentral.com/submit
Biomed Central 